\title{
Su-Schrieffer-Heeger model applied to chains of finite length
}

\author{
Fernando L. J. Vos, Daniel P. Aalberts, and Wim van Saarloos \\ Instituut-Lorentz for Theoretical Physics, Leiden University, P. O. Box 9506, 2300 RA Leiden, The Netherlands
}

(Received 2 November 1995)

\begin{abstract}
We discuss both the ground-state properties and the kink-antikink dynamics of finite conjugated chains, using the Su-Schrieffer-Heeger Hamiltonian with a boundary term added. We establish a clear relationship between model parameters for the case of infinite chains or rings, where one uses periodic boundary conditions, and the case of finite chains for which open boundary conditions are employed. Furthermore, we derive the exact expression for the sound velocity renormalization due to the $\pi$-electron-phonon coupling, arrived at earlier heuristically. The suppression of the sound velocity is only exponentially small in the weak-coupling limit. Some numerical studies of the influence of finite chain length and end effects on kink-antikink dynamics are also presented. [S0163-1829(96)03921-5]
\end{abstract}

\section{INTRODUCTION}

The Su-Schrieffer-Heeger (SSH) Hamiltonian has proven to be a successful theoretical framework for understanding conjugated polymer chains. ${ }^{1-5}$ In this tight-binding model one focuses on the coupling between the $\pi$ electrons that constitute the valence band and the ionic motions along the one-dimensional polymeric chain. As is well known, this model exhibits a rich variety of nonlinear phenomena and topological excitations coupling the two possible and equivalent configurations of bond-length alternation in the Peierls distorted ground-state.

The semiclassical dynamics following the excitation of a $\pi$ electron from the top of the valence band into the bottom of the conduction band in the dimerized ground state has been the subject of a number of papers. ${ }^{6-9}$ However, in these works kink-antikink generation and their dynamics were considered on chains of effectively infinite length only, using periodic boundary conditions; therefore, little is known about finite-size effects. ${ }^{10}$

Our motivation for studying these kink-antikink excitations on chains of finite length comes from a somewhat unexpected corner. In biochemistry one encounters small lightharvesting molecules or "chromophores" that trigger a (not yet fully determined) sequence of steps after photoexcitation. A specific example of such a chromophore is the relatively small conjugated molecule 11-cis-retinal that has a carbon backbone of five $(C-C=C)$ units, and which is bound inside the protein opsin to form the light-sensitive rhodopsin. Rhodopsin is present in membranes of the rod cells of vertebrate retina, thereby enabling perhaps the most important sense: vision.

In recent years (bio)chemists have been slowly uncovering the secrets of vision and now some aspects of the first steps in vision seem well established. To be more specific, photoexcitation of this chromophore leads to an intermediate state (which is called bathorhodopsin) on an extremely short time scale, of the order of $200 \mathrm{fs} .{ }^{11}$ On this time scale the chromophore undergoes a cis-to-trans isomerization; all other processes, which eventually lead to the triggering of a nerve signal, happen on much longer time scales. The first step in vision, the cis-to-trans isomerization of the retinal, therefore appears to be isolated from many of the other biophysical processes that play a role in vision, and presents a challenge to our understanding. Besides being extremely fast - the fastest photochemical reaction - the first step is also found to have a high quantum yield of about $65 \%$, meaning that for every 100 photons supplied, 65 bathorhodopsin molecules are formed. These two remarkable facts, the speed and the efficiency of the first step in vision, lead us to believe that the physical principles involved are due to classical-coherent motion of the elementary excitations.

To study this system theoretically, one has to come up with a definite model. Because of the fact that many details of the structure and function of rhodopsin are not yet known and that it is unclear precisely which details are relevant to the functioning of rhodopsin, a complete model obviously is asking too much. It does seem clear, however, that an extension of the SSH model (taking into consideration torsional degrees of freedom) is well suited because of the fact that the chromophore itself is a small conjugated molecule. The SSH model is also a model of intrinsic simplicity and one in which kink-antikink excitations are consistent with both the short time scale and the high quantum yield. In fact there are experimental indications that the charge distributions in the neighborhood of a charged nitrogen group on the retinal are described quite well by the SSH Hamiltonian with Coulomb corrections. ${ }^{12}$ In our opinion, studying the effects of finite chain lengths on the kink-antikink dynamics within the $\mathrm{SSH}$ model is a modest but logical first step towards the understanding of the first step in vision.

Before we can turn our attention to the dynamics of the untwisting of the retinal, it is necessary to formulate more precisely how to study chains of finite length within the framework of a SSH-type model. It is this issue which is the subject of this article. In order to study chains of finite length without periodic boundary conditions, the question arises as to which boundary condition to use, e.g., whether to leave the chain ends open or to use a potential at the outer ends to regulate the chain length. Although this question has arisen before, it has, to our knowledge, not been addressed systematically. We do so in this paper, and in particular we calculate the value of the stretching force which facilitates comparison between long chains with nonperiodic boundary 
conditions and those with periodic boundary conditions.

Our analysis relies on a general expression that we derive for the energy per site $\varepsilon(u, \delta)$ of the SSH model, for uniform but arbitrary values of the dimerization amplitude $u$ and bond stretching $\delta$. We show that a careful but relatively straightforward evaluation of $\varepsilon(u, \delta)$ for a finite and open SSH chain allows one to determine the proper boundary conditions such that the bulk properties (ground-state dimerization amplitude $\underline{u}$ and stretching $\underline{\delta}$ ) of long but open SSH chains are the same as those of periodic chains for the same parameter sets. This facilitates comparison of results for the two types of boundary conditions.

In fact, the central role played by $\varepsilon(u, \delta)$ for longwavelength properties was already demonstrated by us ${ }^{13}$ recently in another context: In the SSH model, a longwavelength acoustic mode corresponds to a gradual change in $\delta$, and the optical mode to one in $u$; so the second derivatives $\varepsilon_{\delta \delta}$, etc., play the roles of elastic coefficients. This allows one to derive a compact exact expression for the sound velocity in the SSH model,

$$
c=c_{0} \sqrt{\frac{\varepsilon_{\delta \delta}}{K}-\frac{\varepsilon_{u \delta}^{2}}{K \varepsilon_{u u}}},
$$

where $c_{0}$ is the sound velocity in the absence of $\pi$-electron-phonon coupling, and $K$ the bare elastic constant [defined in Eq. (4) below]. As we only gave a physically motivated but heuristic derivation of Eq. (1) in Ref. 13, we give its explicit derivation from the equations of motion in this paper. For a discussion of the implications of Eq. (1), in particular the fact that the sound velocity renormalization is exponentially small for weak coupling, we refer the reader to Ref. 13.

In Sec. II we present the SSH model, discuss the boundary conditions, and show which choice of a stretching force is most convenient to compare various boundary conditions. We then derive Eq. (1) in Sec. III. In Sec. IV we briefly discuss the generation and subsequent dynamics of kinkantikink pairs on finite chains, and compare it to the the case of these excitations on a chain of infinite length or periodic chains. Finally, in Sec. V, we summarize our findings and pose some questions for future study.

\section{MODEL HAMILTONIAN FOR FINITE CHAINS}

The one-dimensional tight-binding Hamiltonian we use to describe the physics of the conjugated polymer transpolyacetylene $(\mathrm{CH})_{N}$, is given by

$$
H=H_{\mathrm{el}}+H_{l},
$$

with the $\pi$-electron-lattice coupling written as

$$
H_{\mathrm{el}}=-\sum_{s} \sum_{n=1}^{N-1}\left[t-\alpha\left(u_{n+1}-u_{n}\right)\right]\left[c_{n, s}^{\dagger} c_{n+1, s}+\text { H.c. }\right]
$$

and a lattice part

$$
H_{l}=\frac{K}{2} \sum_{n=1}^{N-1}\left(u_{n+1}-u_{n}\right)^{2}+\frac{M}{2} \sum_{n=1}^{N} \dot{u}_{n}^{2}-\Gamma \sum_{n=1}^{N-1}\left(u_{n+1}-u_{n}\right) .
$$

In Eqs. (3) and (4), $n$ numbers the $(\mathrm{CH})$ groups, $u_{n}$ is the displacement along the chain of the $n$th $(\mathrm{CH})$ group relative to some reference position $n a$, and $c_{n, s}^{\dagger}\left(c_{n, s}\right)$ creates (annihilates) an electron with spin projection $s$ at site $n$. The model parameters are the hopping parameter $t$ for uniform spacing $a$ between adjacent $(\mathrm{CH})$ groups, the electronphonon coupling constant $\alpha$, the force constant $K$ for bondlength deviations from equal spacing of the $\sigma$-bonding backbone, and the mass of a $(\mathrm{CH})$ group $M$. The harmonic stretching force $\Gamma$ will be discussed below.

The $\pi$-electron-lattice part of the Hamiltonian $H_{\mathrm{el}}$ models the coupling of the $\pi$ electrons to the lattice degrees of freedom via a linear (distance) modulation of the bare hopping frequency $t$. The first term in the lattice part of the Hamiltonian $H_{l}$ models a harmonic restoring force on the $\sigma$-bonded $(\mathrm{CH})$ groups when displaced from equal spacing $a$, and the second term is the kinetic energy. Up to the last term in Eq. (4) the three equations constitute the familiar SSH Hamiltonian. ${ }^{1,2}$

The last term in Eq. (4) gives a constant stretching force $\Gamma$ on a finite chain. As $\Sigma_{n}^{N-1}\left(u_{n+1}-u_{n}\right)=\left(u_{N}-u_{1}\right)$ denotes the change of length of the chain, it corresponds to a potential term which is linear in the total chain length. Usually, the SSH model is studied with periodic boundary conditions, as these are most convenient to model long, essentially infinite chains. As already recognized by Vanderbilt and Mele ${ }^{14}$ and by $\mathrm{Su}^{15}$ however, for finite open chains, which are our interest here, the electronic energy decreases with an overall contraction of the chain due to the linear coupling term proportional to $\alpha$ in $H_{\mathrm{el}}$. Following these authors, a constant stretching force $\Gamma$ is introduced in the Hamiltonian to counterbalance this compression. With this procedure, one can use the same parameters $t, K$, and $\alpha$ as in the model with periodic boundary conditions. Note that for periodic boundary conditions this term automatically vanishes, since then $\left(u_{N}-u_{1}\right)=0$.

At this point, we note that for finite chains without periodic boundary conditions, two types of boundary conditions have been used: so-called "pressure boundary conditions", with $\Gamma \neq 0$ and "open boundary conditions", with $\Gamma=0 .{ }^{16} \mathrm{It}$ is important to realize, however, that from the point of view of using the SSH model Hamiltonian as an effective model, both cases describe the same physics: The "pressure boundary conditions" can be transformed into "open boundary conditions" by a redefinition of the variables $\left\{u_{n}\right\}$ and the parameters $t$ and $\Gamma$. Indeed, under the uniform stretching transformation $\widetilde{u_{n}}=u_{n}-n \Gamma / K$, we find from Eqs. (2) and (4) that to within a constant term

$$
H\left(\left\{u_{n}\right\} ; t, K, \Gamma\right)=H\left(\left\{\tilde{u}_{n}\right\} ; t-\alpha \Gamma / K, K, 0\right) .
$$

Hence, contrary to what is sometimes suggested in the literature, ${ }^{16}$ the dynamics of a chain with pressure boundary conditions is completely equivalent to that of a chain with open boundary conditions, provided we use the renormalized hopping frequency $\widetilde{t}=t-\alpha \Gamma / K$ and uniformly scaled coordinates. ${ }^{15}$ The practical advantage of using the pressure boundary condition with $\Gamma \neq 0$, however, is that with a proper choice of $\Gamma$, we may use the same parameter sets and 
lattice spacing as those used in the literature for periodic chains. These sets were obtained by comparison with experimental data on polyacetylene.

Following Vanderbilt and Mele, ${ }^{14}$ the value $\Gamma=4 \alpha / \pi$ has often been used in the literature. This is the value derived assuming the ground state is undimerized, but in practice a somewhat different value for $\Gamma$ must be used to obtain the proper dimerized ground state. For small coupling, when the changes in the electronic energies due to the dimerization are exponentially small, the correction to $\Gamma=4 \alpha / \pi$ is also exponentially small.

In this section, we shall determine the value of $\Gamma$ selfconsistently for the dimerized ground state of long chains; as we shall see, for the standard parameter sets, the corrections are non-negligible. In addition, the analysis given below will allow us to determine the ground-state energy per site $\varepsilon(u, \delta)$ as a function of the uniform dimerization amplitude $u$ and the uniform bond stretching $\delta$. In Sec. III we show that the optical frequency and sound velocity can be expressed simply in terms of derivatives of $\varepsilon(u, \delta)$. As noted before, ${ }^{13}$ this yields a physically transparent and technically efficient way of calculating the sound velocity exactly.

To obtain the approximate ground state we take $u_{n}$ to be of the form

$$
u_{n}=(-1)^{n} u-\left(\frac{N}{2}-n\right) \delta,
$$

where $N$ is the total number of $(\mathrm{CH})$ groups. On substitution of Eq. (6) and neglecting nonextensive terms, the Hamiltonian Eq. (2) becomes ${ }^{17}$

$$
\begin{aligned}
H(u, \delta)= & -\sum_{n, s}\left[t+2 \alpha(-1)^{n} u-\alpha \delta\right]\left[c_{n, s}^{\dagger} c_{n+1, s}+\text { H.c. }\right] \\
& +2 N K u^{2}+\frac{1}{2} N K \delta^{2}-N \Gamma \delta .
\end{aligned}
$$

The diagonalization of Eq. (7) can be done in analogy with the usual case of periodic boundary conditions, ${ }^{3}$ and so we will only give some of the essential steps. Since we neglect end effects, our results give the dominant term for $\Gamma$ and $\varepsilon(u, \delta)$ in the limit $N \rightarrow \infty$.

For $\alpha=0, H(u, \delta)$ can be brought to diagonal form by the Bloch operators $c_{k s}=N^{-1 / 2} \sum e^{-i k n a} c_{n s}$ in the extended zone $-\pi<k a<\pi$. For $\alpha \neq 0$, when the dimerization doubles the unit cell, it is convenient to fold the zone into the half zone $-\pi / 2<k a<\pi / 2$, with valence $(-)$ and conduction $(+)$ band operators defined as

$$
\begin{gathered}
c_{k s-}=\frac{1}{\sqrt{N}} \sum_{n} e^{-i k n a} c_{n s}, \\
c_{k s+}=\frac{-i}{\sqrt{N}} \sum_{n} e^{-i k n a}(-1)^{n} c_{n s} .
\end{gathered}
$$

In terms of these operators the Hamiltonian is written as

$$
\begin{aligned}
H(u, \delta)= & \sum_{k s}\left[\epsilon_{k}\left(c_{k s+}^{\dagger} c_{k s+}-c_{k s-}^{\dagger} c_{k s-}\right)+\Delta_{k}\left(c_{k s+}^{\dagger} c_{k s-}\right.\right. \\
& \left.\left.+c_{k s-}^{\dagger} c_{k s+}\right)\right]+2 N K u^{2}+\frac{1}{2} N K \delta^{2}-N \Gamma \delta
\end{aligned}
$$

with the energy gap parameter $\Delta_{k}=4 \alpha u \sin (k a)$ and unperturbed band energy in the reduced zone defined by

$$
\epsilon_{k}=2(t-\alpha \delta) \cos (k a)
$$

Finally, $H$ is diagonalized by the transformations $a_{k s-}=\beta_{k} c_{k s-}-\gamma_{k} c_{k s+}$ and $a_{k s+}=\beta_{k} c_{k s-}+\gamma_{k} c_{k s+}$, whose inverses, on substitution in Eq. (9), give

$$
\begin{aligned}
H(u, \delta)= & \sum_{k s} E_{k}\left(n_{k s+}-n_{k s-}\right)+2 N K u^{2} \\
& +\frac{1}{2} N K \delta^{2}-N \Gamma \delta,
\end{aligned}
$$

with the quasiparticle energy of the familiar form $E_{k}=\sqrt{\epsilon_{k}^{2}+\Delta_{k}^{2}}$ and $n_{k s \pm}=a_{k s \pm}^{\dagger} a_{k s \pm}$. Note that since the $\epsilon_{k}$ and hence $E_{k}$ depend on the bond stretching $\delta$ according to Eq. (10), the first term of the right-hand side of Eq. (11) depends on $\delta$ as well.

For the half-filled band of $(\mathrm{CH})_{N}$, the energy per site $\varepsilon(u, \delta)$ for a given dimerization amplitude $u$ and stretch $\delta$ is obtained by setting $n_{k s-}=1$ and $n_{k s+}=0$ in Eq. (11), and replacing the sum by an integral:

$$
\begin{aligned}
\varepsilon(u, \delta)= & \frac{-2}{\pi} \int_{0}^{\pi / 2} E_{k} d(k a)+2 K u^{2}+\frac{1}{2} K \delta^{2}-\Gamma \delta \\
= & \frac{-4(t-\alpha \delta)}{\pi} \mathscr{E}\left(\sqrt{1-z^{2}}\right) \\
& +2 K u^{2}+\frac{1}{2} K \delta^{2}-\Gamma \delta,
\end{aligned}
$$

where we have introduced the dimensionless variable $z$, given by

$$
z=\frac{2 \alpha u}{t-\alpha \delta}
$$

and where $\mathscr{E}$ is the complete elliptic function of the second kind:

$$
\mathscr{E}\left(\sqrt{1-z^{2}}\right)=\int_{0}^{\pi / 2} \sqrt{1-\left(1-z^{2}\right) \sin ^{2}(\phi)} d \phi .
$$

From Eq. (12) we can determine the ground-state dimerization amplitude and uniform stretch for our chains by minimization of the energy. Taking first derivatives with respect to $u$ and $\delta$ yields

$$
\begin{gathered}
\frac{\partial \varepsilon(u, \delta)}{\partial u}=\frac{8 \alpha}{\pi} \frac{z}{1-z^{2}}[\mathscr{E}-\mathscr{K}]+4 K u, \\
\frac{\partial \varepsilon(u, \delta)}{\partial \delta}=\frac{4 \alpha}{\pi} \frac{1}{1-z^{2}}\left[\mathscr{E}-z^{2} \mathscr{K}\right]+K \delta-\Gamma,
\end{gathered}
$$

where $\mathscr{K}$ is the complete elliptic integral of the first kind,

$$
\mathscr{K}\left(\sqrt{1-z^{2}}\right)=\int_{0}^{\pi / 2} \frac{1}{\sqrt{1-\left(1-z^{2}\right) \sin ^{2}(\phi)}} d \phi,
$$

and where we have begun to abbreviate $\mathscr{E}\left(\sqrt{1-z^{2}}\right)$ and $\mathscr{K}\left(\sqrt{1-z^{2}}\right)$ as $\mathscr{E}$ and $\mathscr{K}$. 


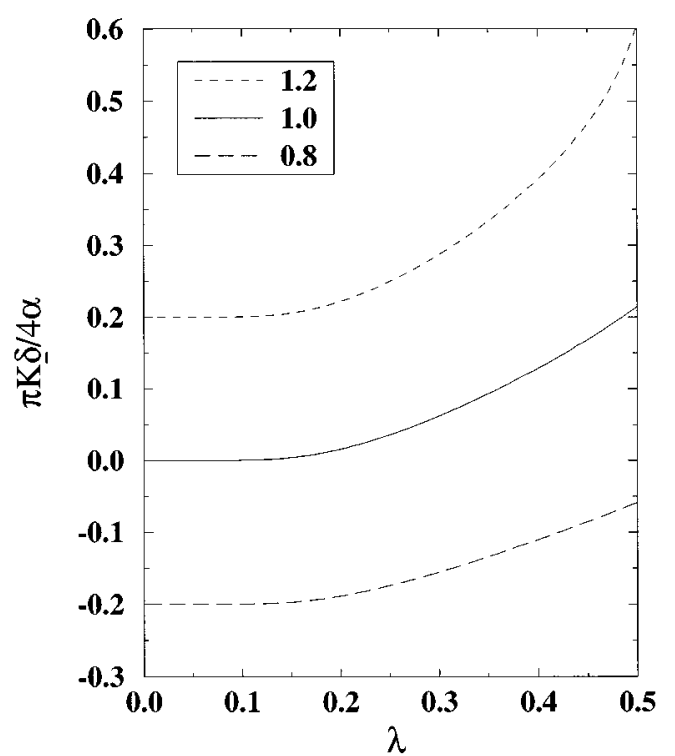

FIG. 1. The uniform stretch per bond $\delta$ as a function of coupling strength $\lambda$, for $\Gamma /(4 \alpha / \pi)=0.8,1.0$, and 1.2.

By differentiating Eqs. (15) and (16) once more, we get for the second derivatives of $\varepsilon$ with respect to $u$ and $\delta$

$$
\begin{gathered}
\varepsilon_{u u}=\frac{16 \alpha^{2}}{\pi(t-\alpha \delta)} \frac{2 \mathscr{E}-\left(1+z^{2}\right) \mathscr{K}}{\left(1-z^{2}\right)^{2}}+4 K, \\
\varepsilon_{\delta \delta}=\frac{4 \alpha^{2} z^{2}}{\pi(t-\alpha \delta)} \frac{2 \mathscr{E}-\left(1+z^{2}\right) \mathscr{R}}{\left(1-z^{2}\right)^{2}}+K, \\
\varepsilon_{u \delta}=\frac{8 \alpha^{2} z}{\pi(t-\alpha \delta)} \frac{2 \mathscr{E}-\left(1+z^{2}\right) \mathscr{K}}{\left(1-z^{2}\right)^{2}} .
\end{gathered}
$$

Together with Eq. (1), these equations give the explicit expressions for the speed of sound.

The ground-state configuration can now be determined by setting the first derivatives Eqs. (15) and (16) to zero and solving for $\underline{u}$ and $\underline{\delta}$ as a function of the model parameters. It is convenient to introduce a dimensionless electron-lattice coupling strength $\lambda$, which is defined here as ${ }^{18}$

$$
\lambda=\frac{2 \alpha^{2}}{\pi K t} .
$$

For the stretch per bond $\delta$ in the ground-state and the parameter $z$ defined in Eq. (13), we obtain from Eqs. (15) and (16) the two coupled equations

$$
\begin{gathered}
\frac{\pi K}{4 \alpha} \underline{\delta}=\frac{1}{2 \lambda}+\frac{\mathscr{E}-\mathscr{K}}{1-z^{2}}, \\
\frac{1}{2 \lambda}=\frac{\pi \Gamma}{4 \alpha}-\frac{2 \mathscr{E}-\left(1+z^{2}\right) \mathscr{K}}{1-z^{2}} .
\end{gathered}
$$

These coupled equations can be solved numerically; i.e., given $\Gamma$ and $\lambda$ one determines $z$ from Eq. (22b), thus giving the (scaled) stretch $\pi K \underline{\delta} / 4 \alpha$ on substitution in Eq. (22a). Figure 1 shows $\underline{\delta}$ as a function of the coupling strength $\lambda$ for different values of $\Gamma$.

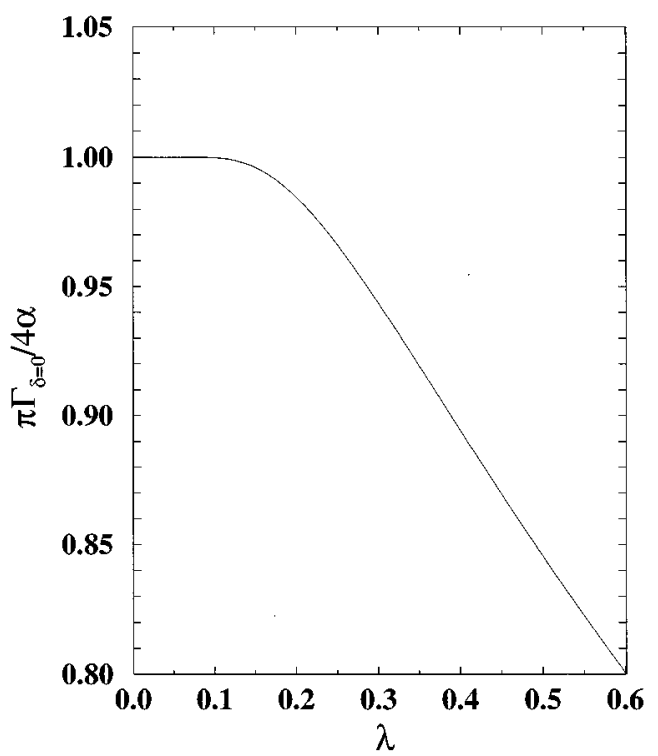

FIG. 2. Value of $\Gamma$ that renders $\delta=0$ in the ground state versus coupling strength $\lambda$.

As we mentioned previously, we want to tune the parameter $\Gamma$ in such a way that there is no stretching $(\underline{\delta}=0)$ in the ground-state, as in the case of periodic boundary conditions. This value of $\Gamma$, where no stretching occurs, is obtained by solving the set of coupled equations

$$
\begin{gathered}
\frac{1}{2 \lambda}=\frac{\mathscr{K}-\mathscr{E}}{1-z^{2}}, \\
\Gamma_{\delta=0}=\frac{4 \alpha}{\pi} \frac{\mathscr{E}-z^{2} \mathscr{K}}{1-z^{2}} .
\end{gathered}
$$

Figure 2 depicts the dependence of $\pi \Gamma_{\delta=0} / 4 \alpha$ on the coupling strength $\lambda$. The weak-coupling correction to $\Gamma_{\delta=0}$ is only exponentially small; for small $\lambda$, we obtain from Eqs. (23)

$$
\begin{gathered}
z \sim 4 e^{-[1+1 /(2 \lambda)]}, \\
\frac{\pi \Gamma_{\delta=0}}{4 \alpha} \sim 1-4\left(\frac{1}{\lambda}-1\right) e^{-(2+1 / \lambda)} .
\end{gathered}
$$

The actual values of the parameters for polyacetylene depend on the type of experiment from which they are extracted. ${ }^{5}$ For the different parameter sets used the literature, however, $\lambda$ lies in the range between 0.2 and 0.4 , so that the correction is non-negligible.

\section{SOUND VELOCITY DERIVATION}

As noted before, ${ }^{13}$ the sound velocity $c$ can be expressed simply in terms of the second derivatives of $\varepsilon(u, \delta)$ through Eq. (1). For the undimerized chain, this result follows straightforwardly from the observation that $\varepsilon_{\delta \delta}$ plays the role of the elastic constant that gives the energy change associated with a small uniform stress. The general formula [Eq. (1)] was derived heuristically in Ref. 13, but here we show that this result can be obtained directly from the equations of motion as well. 
As a starting point we take the classical equation of motion for the $j$ th $(\mathrm{CH})$ group, expressed in external coordinates:

$$
M \ddot{x}_{j}=\frac{-\partial E}{\partial x_{j}},
$$

where $E=E\left(\left\{x_{i}\right\}\right)$ is the total energy which, in the adiabatic approximation, depends on the nuclear coordinates only. Changing to internal coordinates with two atoms per unit cell,

$$
\begin{gathered}
\delta_{2 n}=\frac{1}{4}\left(x_{2 n+3}+x_{2 n+2}-x_{2 n+1}-x_{2 n}\right), \\
v_{2 n}=x_{2 n+1}-x_{2 n},
\end{gathered}
$$

we obtain the two coupled equations of motion

$$
\begin{gathered}
-4 M \ddot{\delta}_{2 n}=-\frac{1}{2} \frac{\partial E}{\partial \delta_{2 n-2}}+\frac{\partial E}{\partial \delta_{2 n}}-\frac{1}{2} \frac{\partial E}{\partial \delta_{2 n+2}}, \\
-M \ddot{v}_{2 n}=2 \frac{\partial E}{\partial v_{2 n}} .
\end{gathered}
$$

On introducing the Fourier transforms

$$
\begin{gathered}
\widetilde{\delta}_{q}=\frac{2}{N} \sum_{n=1}^{N / 2} \delta_{2 n} e^{i q(2 a n)}, \\
\frac{\partial}{\partial \widetilde{\delta}_{p}}=\sum_{n=1}^{N / 2} e^{-i p(2 a n)} \frac{\partial}{\partial \delta_{2 n}},
\end{gathered}
$$

and expanding around the first derivatives in Eqs. (28) and (29), the equations of motion become

$$
\begin{aligned}
& -M \ddot{\ddot{\delta}_{q}}=\frac{A(q)}{2 N} \sum_{p}\left[\widetilde{\delta}_{p} \frac{\partial^{2} E}{\partial \widetilde{\delta}_{-q} \partial \widetilde{\delta}_{p}}+\widetilde{v}_{p} \frac{\partial^{2} E}{\partial \widetilde{\delta}_{-q} \partial \widetilde{v}_{p}}\right], \\
& -M \ddot{\tilde{v}_{q}}=\frac{4}{N} \sum_{p}\left[\widetilde{\delta}_{p} \frac{\partial^{2} E}{\partial \widetilde{v}_{-q} \partial \widetilde{\delta}_{p}}+\widetilde{v}_{p} \frac{\partial^{2} E}{\partial \widetilde{v}_{-q} \partial \widetilde{v}_{p}}\right],
\end{aligned}
$$

with $A(q)=1-\cos (2 q a)$. Defining the energy per site $\varepsilon \equiv E / N$, substituting $\left(-i \omega_{q}\right)$ for a time derivative, and taking advantage of translational invariance in the ground state, we find

$$
\begin{gathered}
M \omega_{q}^{2} \widetilde{\delta}_{q}=\frac{A(q)}{2}\left[\widetilde{\delta}_{q} \frac{\partial^{2} \varepsilon}{\partial \widetilde{\delta}_{-q} \partial \widetilde{\delta}_{q}}+\widetilde{v}_{q} \frac{\partial^{2} \varepsilon}{\partial \widetilde{\delta}_{-q} \partial \widetilde{v}_{q}}\right], \\
M \omega_{q}^{2} \widetilde{v}_{q}=4\left[\widetilde{\delta}_{q} \frac{\partial^{2} \varepsilon}{\partial \widetilde{v}_{-q} \partial \widetilde{\delta}_{q}}+\widetilde{v}_{q} \frac{\partial^{2} \varepsilon}{\partial \widetilde{v}_{-q} \partial \widetilde{v}_{q}}\right] .
\end{gathered}
$$

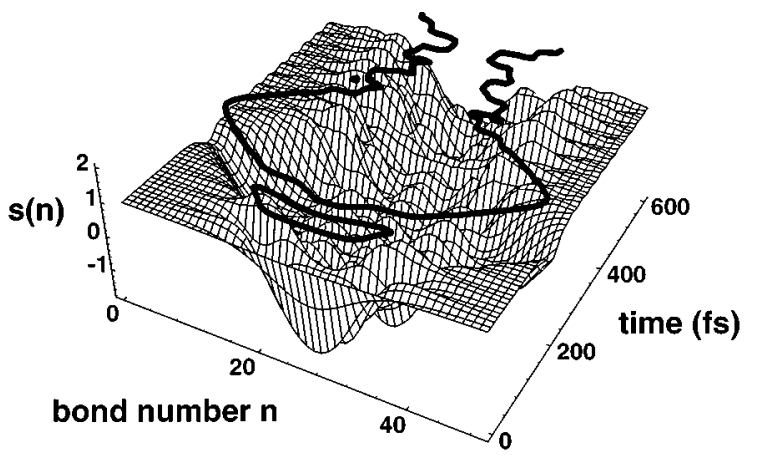

FIG. 3. The kink-antikink dynamics on a $N=50$ chain, with the parameters given in the text. The heavy line denotes the zero crossings of $s(n)$ and shows how a kink, initially moving towards the chain end with an approximately uniform speed, is reflected on approaching the end at a distance $\xi$. It moves back again with approximately uniform speed.

One final change of coordinates to account for the dimerization amplitude $u_{2 n}$ is defined from $v_{2 n}=\delta_{2 n}+2 u_{2 n}$, which means that the derivatives with respect to $u$ (at constant $\delta$ ) are given by

$$
\frac{\partial}{\partial v_{2 n}}=\frac{1}{2} \frac{\partial}{\partial u_{2 n}}
$$

Thus finally we have

$M \omega_{q}^{2}\left(\begin{array}{c}\widetilde{\delta}_{q} \\ \widetilde{v_{q}}\end{array}\right)=\left(\begin{array}{cc}\frac{1}{2} A(q) \widetilde{\varepsilon}_{\delta \delta} & \frac{1}{4} A(q) \widetilde{\varepsilon}_{\delta u} \\ 2 \widetilde{\varepsilon_{u \delta}} & \widetilde{\varepsilon}_{u u}\end{array}\right)\left(\begin{array}{c}\widetilde{\delta}_{q} \\ \widetilde{v_{q}}\end{array}\right)$,

where we have introduced the notation

$$
\widetilde{\varepsilon_{x y}} \equiv \frac{\partial^{2} \varepsilon}{\partial \widetilde{x}_{-q} \partial \widetilde{y}_{q}} .
$$

Therefore, in the long-wavelength limit where $\lim _{q \rightarrow 0} \widetilde{\varepsilon}_{x y}=\varepsilon_{x y}$, we find for the acoustic frequency

$$
\omega=q a \sqrt{\frac{\varepsilon_{\delta \delta}-\varepsilon_{\delta u}^{2} / \varepsilon_{u u}}{M}},
$$

from which we obtain the sound velocity as

$$
c=c_{0} \sqrt{\frac{\varepsilon_{\delta \delta}-\varepsilon_{\delta u}^{2} / \varepsilon_{u u}}{K}},
$$

with $c_{0}=\sqrt{K / M}$, which reproduces Eq. (1). The $q \rightarrow 0$ optical frequency becomes, according to Eq. (37),

$$
\omega_{\mathrm{opt}}=\sqrt{\frac{\varepsilon_{u u}}{M}}
$$

We stress that within the adiabatic approximation Eqs. (40) and (41) are exact. They can be calculated explicitly using 
Eqs. (18)-(20) evaluated at the equilibrium values $\underline{u}$ and $\delta$. For a full discussion of these results we refer to Ref. 13; here we just note that by expanding these equations for small $\lambda$, we find an exponentially small renormalization of the sound velocity for small $\lambda$ :

$$
c / c_{0} \approx 1-4\left(\frac{1}{\lambda}-2\right) e^{-(2+1 / \lambda)},
$$

which invalidates the often quoted result ${ }^{3,4}$ that the sound velocity for small $\lambda$ is given by $c=c_{0} \sqrt{1-2 \lambda}$ and also the result obtained by Rice et al. ${ }^{19}$

The optical frequency can also be explicitly calculated from Eqs. (41) and (18). For weak coupling we obtain

$$
\omega_{\mathrm{opt}} \approx \sqrt{2 \lambda} \Omega_{0},
$$

where $\Omega_{0}=\sqrt{4 K / M}$ would be the $(q=0)$ optical frequency in the absence of $\pi$-electron-phonon coupling. This result and that given in the literature agree. ${ }^{13}$

\section{DYNAMICS}

Since, as explained in the Introduction, our interest ultimately lies in exploring the dynamical pathway to conformational changes in rhodopsin as it adsorbs a photon, we conclude this paper by briefly discussing dynamical simulations of photoexcitations for finite chains.

Our chief aim here is to investigate the effect of the stretching force $\Gamma$ (pressure boundary conditions) on the dynamics of a kink-antikink pair formed when an electron is excited from the top of the valence band into the bottom of the conduction band. In particular, since these solitons are repelled from the ends of a chain, ${ }^{15}$ one expects that dynamically generated solitons will be reflected by the chain ends. The results presented below confirm this expectation, and show that solitons and breathers ${ }^{20,21}$ are still recognizable entities on small chains. ${ }^{10}$

Our simulation technique is based on the adiabatic approximation using the Feynman-Hellmann theorem. ${ }^{22}$ In short, the procedure is to diagonalize the electronic Hamiltonian at every time step, and to calculate the electronic forces on the $(\mathrm{CH})$ groups using the Feynman-Hellmann theorem.

To illustrate the generic dynamics on an open chain of finite length, we present the results we obtained for a chain of $N=50$ sites and parameter values set $t^{20,21} t=2.5 \mathrm{eV}$, $\alpha=4.8 \mathrm{eV} / \AA$, and $K=17.3 \mathrm{eV} / \AA^{2}$. These parameters imply a coupling strength $\lambda=0.34$ from the definition of $\lambda$ in Eq. (21). For the value of $\Gamma_{\delta=0}$, needed to obtain a ground state with zero bond stretch, we find $\Gamma_{\delta=0}=5.648 \mathrm{eV} / \AA$ from Eqs. (23a) and (23b). Furthermore, the electronic length scale $\xi$, which determines the width of a kink, is found to be $\xi / a=(2 t / \Delta) \approx 2.5$.

Figure 3 shows a three-dimensional representation of the dynamics. Along the vertical axis we have plotted the bond elongation relative to its ground-state value, i.e., $s(n) \equiv\left(u_{n+1}-u_{n}\right) /\left(u_{n+1}-u_{n}\right)$. With the heavy line we show the $s(n)=0$ crossing (shifted upwards for better visibility).

Obviously the dynamics on this open chain very much resembles the dynamics on chains with periodic boundary conditions in the first instants. After about 100 fs a kinkantikink pair is clearly formed, moving apart with approximately uniform speed (heavy line). As in periodic chains, a spatially localized oscillating mode or 'breather' is left behind because, as pointed out by Bishop et al., ${ }^{20,21}$ the energy of the two moving kinks is less than the energy injected by creating the electron-hole pair. The surplus energy is radiated backwards by the moving kink and antikink and forms the breather. The kink and antikink continue to move apart with approximately uniform velocity, until they are at a distance of the order $\xi$ from the end. There they bounce back because solitons are repelled from the ends ${ }^{15}$ and move towards the center, again with an approximately uniform speed. Finally, the kink and antikink interact with each other and with the breather in a complicated way; they then reemerge from this zone after about 600 fs. Coulomb interaction of the charged kink and antikink may be important in understanding the eventual relaxation of the molecule to its final state.

For different parameters the length scales and time scales are of course different, but we have found the dynamics described above to be generic. We leave a more systematic study of finite chain dynamics to the future.

\section{SUMMARY AND OUTLOOK}

We have shown how the introduction of an additional degree of freedom, a uniform bond stretch, enables us to apply the SSH model to finite open chains. The advantage of this approach lies in the fact that one can use the same parameter sets for our finite chains as used for infinite chains with periodic boundary conditions.

Both these results and those for the renormalization of the sound velocity are given in terms of the energy per site $\varepsilon(u, \delta)$. Our method is, in fact, completely general in that it can be applied to any model in which an effective energy for the long-wavelength modes can be written down. ${ }^{13}$

The initial (adiabatic) dynamics, following the excitation of an electron from the top of the valence band into the bottom of the conduction band, is qualitatively the same as the dynamics on periodic chains, and confirm that solitons are reflected at the chain ends.

The insights we have obtained in studying the SSH model on finite chains will help us to move on to a more elaborate model for the conformational changes in rhodopsin after photoexcitation. To this end, torsional degrees of freedom and ionic or other site impurities may each play a part. It is our belief that the same classical coherent dynamics as seen in the SSH model plays an essential role in the first step of vision, and preliminary investigations along these lines seem very promising.

\section{ACKNOWLEDGMENTS}

We are grateful to Huub de Groot, Jan Zaanen, and JanAdriaan Leegwater for their interest and many stimulating discussions. The work of F.L.J.V. and D.P.A. is supported by the "Netherlands Foundation for Fundamental Research on Matter", (FOM). 
${ }^{1}$ W.P. Su, J.R. Schrieffer, and A.J. Heeger, Phys. Rev. Lett. 42, 1698 (1979).

${ }^{2}$ W.P. Su, J.R. Schrieffer, and A.J. Heeger, Phys. Rev. B 22, 2099 (1980).

${ }^{3}$ A.J. Heeger, S. Kivelson, J.R. Schrieffer, and W.P. Su, Rev. Mod. Phys. 60, 781 (1988).

${ }^{4}$ Yu Lu, Solitons \& Polarons in Conducting Polymers (World Scientific, Singapore, 1988).

${ }^{5}$ D. Baeriswyl, in Theoretical Aspects of Band Structures and Electronic Properties of Pseudo-One-Dimensional Solids, edited by H. Kamimura (Reidel, Dordrecht, 1985).

${ }^{6}$ W.P. Su and J.R. Schrieffer, Proc. Natl. Acad. Sci. USA 77, 5626 (1980).

${ }^{7}$ E.J. Mele, Solid State Commun. 44 , 827 (1982).

${ }^{8}$ E.J. Mele, Phys. Rev. B 26, 6901 (1982).

${ }^{9}$ F. Guinea, Phys. Rev. B 30, 1884 (1984).

${ }^{10}$ One study of finite molecules is J.L. Brédas and A.J. Heeger, Chem. Phys. Lett. 154, 56 (1989). For short molecules in the first electronically excited state, they study the size dependence of relaxed solitonic steady states. [See also J.L. Brédas, J.M. Toussaint, and A.J. Heeger, Mol. Cryst. Liq. Cryst. 189, 81 (1990).]

${ }^{11}$ R.W. Schoenlein, L.A. Peteanu, R.A. Mathies, and C.V. Shank, Science 254, 412 (1991).
${ }^{12}$ H.J.M. de Groot (unpublished).

${ }^{13}$ F.L.J. Vos, D.P. Aalberts, and W. van Saarloos, Phys. Rev. B 53, R5986 (1996).

${ }^{14}$ D. Vanderbilt and E.J. Mele, Phys. Rev. B 22, 3939 (1980).

${ }^{15}$ W.P. Su, Solid State Commun. 35, 899 (1980).

${ }^{16}$ S.R. Phillpot, D. Baeriswyl, A.R. Bishop, and P.S. Lomdahl, Phys. Rev. B 35, 7533 (1985).

${ }^{17}$ We follow common practice (Ref. 3) in calling Eqs. (7) and (9) Hamiltonians. Strictly speaking, the electronic part is written in the form of a Hamiltonian, but as it does not depend on the individual coordinates of the $\mathrm{CH}$-groups anymore, the lattice part in Eq. (9) is not a proper Hamiltonian.

${ }^{18}$ Note that there are several conventions for the definition of the coupling strength $\lambda$ in the literature, mostly differing by factors of 2 .

${ }^{19}$ M.J. Rice, S.R. Phillpot, A.R. Bishop, and D.K. Campbell, Phys. Rev. B 34, 4139 (1986).

${ }^{20}$ A.R. Bishop, D.K. Campbell, P.S. Lomdahl, B. Horovitz, and S.R. Phillpot, Phys. Rev. Lett. 52, 671 (1984).

${ }^{21}$ A.R. Bishop, D.K. Campbell, P.S. Lomdahl, B. Horovitz, and S.R. Phillpot, Synth. Met. 9, 223 (1984).

${ }^{22}$ C. Cohen-Tannoudji, B. Diu, and F. Laloë, Quantum Mechanics (Wiley-Interscience, London, 1991), Vol. 2, p. 1192. 